\title{
The Effect of Disability on the Diagnosis and Treatment of Multiple Myeloma in Korea: A National Cohort Study
}

\author{
Jihyun Kwon, MD' \\ So Young Kim, MD, MPH, PhD',3,4 \\ Kyoung Eun Yeob, MPH, PhD 3 \\ Hye Sook Han, MD, PhD' \\ Ki Hyeong Lee, MD, PhD \\ Dong Wook Shin, MD, DrPH5,6 \\ Yeon-Yong Kim, $\mathrm{MD}^{7}$ \\ Jong Heon Park, MD, PhD ${ }^{7}$ \\ Jong Hyock Park, MD, MPH, PhD ${ }^{3,4}$
}

\begin{abstract}
Purpose
This study aimed to determine whether the diagnosis, treatment approach, and prognosis of multiple myeloma (MM) vary according to the presence and type of disability.

\section{Materials and Methods}

Demographic, socioeconomic, and medical data were obtained from the National Disability Database, the Korean Central Cancer Registry, and the Korean National Health Insurance claims database. An age- and sex-matched cohort was established using a 1:3 ratio constituted with 2,776,450 people with disabilities and 8,329,350 people without disabilities. Adult patients diagnosed with MM were subsequently selected from this cohort. Disabilities were categorized as physical, communication, intellectual or psychological, and affecting the major internal organs.
\end{abstract}

\section{Results}

The cohort included 4,090 patients with MM, with a significantly lower rate per 100,000 persons among people with disabilities than among people without disabilities (29.1 vs. $39.4, p<0.001$ ). People with disabilities were more likely to undergo dialysis treatment at the time of diagnosis (16.3\% vs. $10.0 \%, p<0.001$ ), but were less likely to undergo autologous stem cell transplantation ( $37.5 \%$ vs. $43.7 \%, p=0.072$ ). This trend was more evident among patients with intellectual or psychological disabilities. The median overall survival among patients with disabilities was significantly shorter than that among patients without disabilities (36.8 months vs. 51.2 months, $p<0.001$ ).

\section{Conclusion}

In Korea, people with disabilities generally have a lower rate of MM diagnosis, receive less intensive treatment, and have a lower survival rate than people without disabilities.

\section{Key words}

Disability, Multiple myeloma, Cohort, Survival
Correspondence: Jong Hyock Park, MD, MPH, PhD College of Medicine/Graduate School of Health Science Business Convergence, Chungbuk National University, 1 Chungdae-ro, Seowon-gu, Cheongju 28644, Korea Tel: 82-43-261-2873

Fax: 82-43-261-3459

E-mail: jonghyock@gmail.com

Received December 19, 2018

Accepted April 21, 2019

Published Online April 22, 2019

*Jihyun Kwon and So Young Kim contributed equally to this work. 


\section{Introduction}

Multiple myeloma (MM), a malignant neoplasm that originates from plasma cells, is the third most common hematological malignancy in Korea with a steadily increasing incidence in recent years [1,2]. The 1990s brought a major change in the treatment strategy for MM, which involved the introduction of high-dose melphalan chemotherapy followed by autologous hematopoietic stem cell transplantation (ASCT). More recent developments have led to the introduction of 'novel agents', such as immunomodulatory drugs and proteasome inhibitors, which have greatly improved the prognosis of patients with MM [3-5]. However, patients must be in relatively good health to undergo an intensive treatment, such as ASCT. In addition, the high economic cost of novel agents also limits access to this treatment. It is unclear whether physically or economically vulnerable populations, such as people with disabilities, are experiencing equal benefits from the development of newer MM therapies.

To date, there is no reported clinical study regarding the effect of a disability on the clinical management and prognosis of hematological cancers, including MM. Korea has various national databases that can provide information regarding different health status factors, such as disability status, cancer, the delivery of healthcare, and associated medical costs. The present study aimed to evaluate differences in the diagnoses, treatment approaches, and outcomes among patients with $\mathrm{MM}$ according to their disability status. Also studied were the effects of the severity and type of disability on the clinical course of MM.

\section{Materials and Methods}

\section{Data sources and case selection}

The present study obtained data from the Korean National Disability Database, the Korean Central Cancer Registry (KCCR), and the Korean National Health Insurance (KNHI) claims database. We identified 2,776,450 persons with disabilities during 2009-2013 using the National Disability Registry, which contains information regarding disability types and severity levels. That database is assumed to have covered $93.8 \%$ of the total population with disabilities in 2011 [6]. Information from the National Disability Registry was merged with the data from the KCCR and the KNHI claims database during 2002-2015 using personal identification numbers. The KCCR is a nationwide government-sponsored cancer registry that is estimated to have covered $97.2 \%$ of cancer cases in 2009 [7]. The KCCR includes the age at diagnosis, date of diagnosis, cancer site, and the Surveillance, Epidemiology, and End Results (SEER) summary stage. The KNHI database has been used extensively for epidemiological and health policy studies [8-10]. The KNHI provides comprehensive universal health insurance to all Koreans and the KNHI database contains information regarding the patients' sex, age, health insurance premium, residential area, comorbid diseases, diagnosis, imaging and laboratory test results, and treatment. The KNHI claims database also contains data on the date of death, which is based on the date of the death declaration provided by the Ministry of Public Administration and Security and which has been partially corrected using the date of the loss of health insurance qualification.

An age and sex-matched cohort was created using a casecontrol match 1:3 ratio consisting of 2,776,450 people with disabilities and 8,329,350 people without disabilities. We subsequently identified 4,358 patients who were diagnosed with $\mathrm{MM}$ and then excluded patients who were $<19$ years old $(\mathrm{n}=0)$, had no information regarding health insurance premiums $(n=23)$, had a diagnosis of other non-thyroid cancer $(n=202)$, or were diagnosed with MM before $2002(n=43)$, as the KNHI only began collecting claims data in 2002.

Among the eligible patients, we identified 496 patients who developed disabilities after their diagnosis of MM. In this group, the rate of major internal organ disability was $36.9 \%$ (180 patients), which was significantly higher than in the entire disability cohort (5.4\%) or the cohort of people with disabilities before MM diagnosis (8.9\%). The most common cause of their disability was renal failure (169 patients), which is a well-known complication of MM. In addition, the physical disability rate was lower at $248(50.0 \%)$ out of 496 patients than in the entire cohort of disability $(62.3 \%)$ and the cohort of people with disabilities before MM diagnosis $(62.5 \%)$, but half of them (123 patients) had disabilities related to spinal problem. Paralysis and weakness caused by spinal cord injury is also a common result of pathological vertebral fracture by MM. In summary, more than $60 \%$ of the 496 patients were found to have a disability associated with MM complications. Therefore, we integrated these 496 patients into the non-disability group.

Finally, the study sample included 809 patients with disabilities upon their MM diagnosis and 3,281 patients without disabilities upon the MM diagnosis (Fig. 1). The patients' personal identification numbers were used to merge their data, although anonymized identifiers were subsequently used during the analysis. 


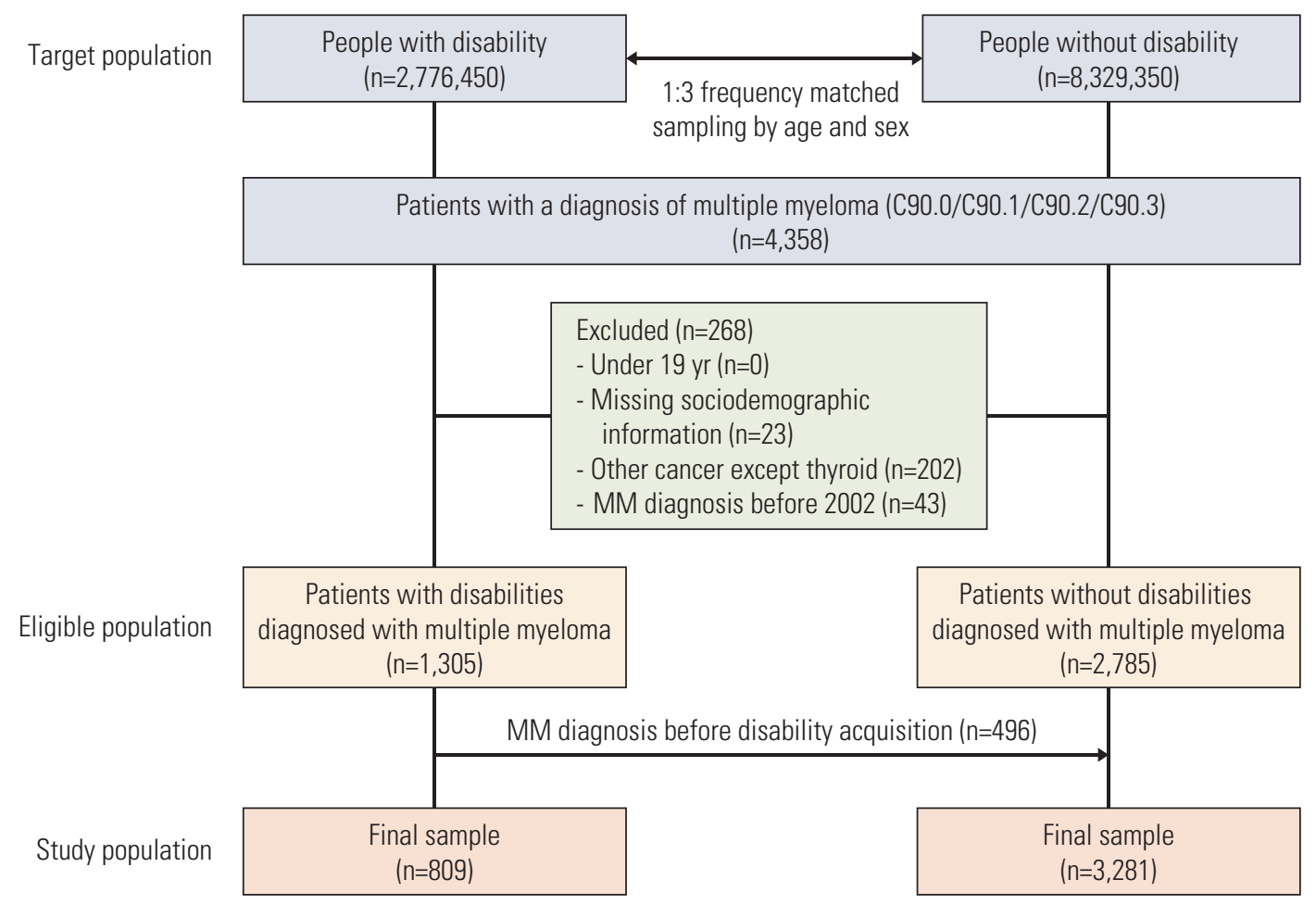

Fig. 1. CONSORT diagram. The total number of multiple myeloma (MM) patients who met the inclusion criteria was 4,090. Except the 496 patients who acquired after being diagnosed with MM, the final number of MM patients with disabilities was 809 . The number of MM patients without disabilities was 3,281.

\section{Variables}

Fifteen disability grouping are included in the National Disability Database [11], which were categorized for the present study as physical, communication, intellectual or psychological, and affecting the major internal organs (S1 Table). Disability severity is graded from 1 (very severe) to 6 (very mild) based on functional losses and clinical impairment as determined by a medical specialist. In the present study, disability severity was classified as severe (grade 1-3) or mild (grade 4-6).

For each patient, cancer-related information was collected from the KCCR, which included the patient's age at diagnosis ( $<65$ years or $\geq 65$ years), date of diagnosis, cancer site, and SEER summary stage. Patient incomes were used to identify patients in the insurance premium categories of below the poverty line (lowest) and quintiles I, II, III, and IV (highest), as provided by the KNHI. The KNHI premium was used to estimate household income because it is calculated based on income, property, and automobile taxes for each household [12].

\section{Outcomes and statistical analyses}

Descriptive analyses were performed to determine the distributions of patients with and without disabilities according to age, sex, and income level in the overall cohort and among patients with MM. The chi-square test was used for these categorical variables. The incidence of major complications, such as pathologic fractures or renal failures at the time of MM diagnosis were also calculated, based on claims that were made from six months before the MM diagnosis to one month after the MM diagnosis. The rates of using ASCT, thalidomide, and bortezomib to treat MM were also analyzed.

All patients were followed until death or 31 December 2017. The survival outcomes and related risk factors were evaluated using the Kaplan-Meier method with the log-rank test and a multivariate Cox proportional hazards regression analysis. Before the analysis, the assumption of proportionality was confirmed by plotting the log hazard estimates against the observation periods. Each covariate that was used for adjustment had a Schoenfeld residual that indicated whether the proportional-hazard assumption was fulfilled ( $p>0.1$ for all covariates). All statistical analyses were per- 


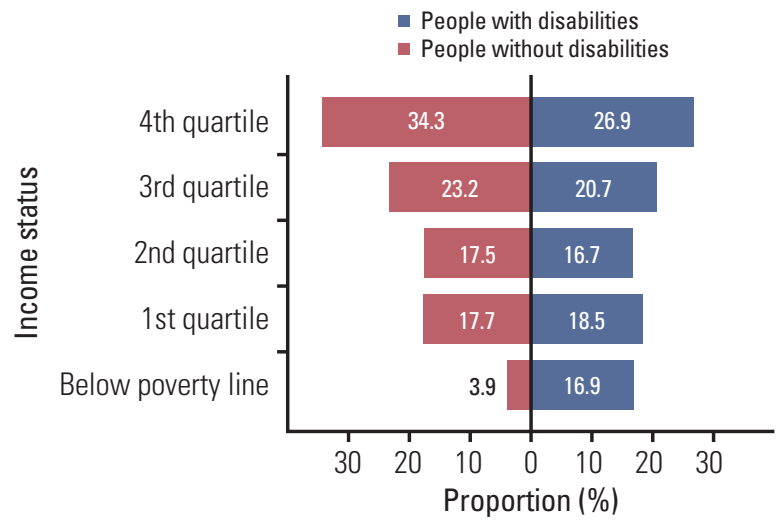

Fig. 2. Comparison of income level structure with or without disability. A comparison of the economic situation of people without disabilities (red) or with disabilities (blue). People with disabilities have a high percentage of below poverty line.

formed using SAS software ver. 9.4 (SAS Institute, Inc., Cary, $\mathrm{NC}$ ) and differences were considered statistically significant at two-sided p-values of $\leq 0.05$.

\section{Ethical statement}

The study protocol was approved by the Institutional Review Board of Chungbuk National University (CBNU201607-BM-288-01).

\section{Results}

\section{Baseline characteristics of the cohorts}

Basic information of the entire cohort is provided in S2 Table. Among the entire cohort, $64.5 \%$ of people were $<65$ years old and $32.1 \%$ of people were $\geq 65$ years old, with $58.5 \%$ of people being male and $41.5 \%$ being female. The proportions of people below the poverty line were $7.1 \%$ in the entire cohort, $16.9 \%$ in the disabled group, and $3.9 \%$ in the non-disabled group, with the disabled group having a significantly lower income level than the non-disabled group (Fig. 2).

The severity and type of disability were also analyzed, which revealing that 1,091,764 people (39.3\%) were severely disabled and 1,684,656 people $(60.7 \%)$ were mildly disabled. Physical disabilities were the most common type $(n=1,728,916$,

Table 1. Baseline characteristics of the patients with MM

\begin{tabular}{|c|c|c|c|c|c|c|c|}
\hline & \multicolumn{2}{|c|}{ Entire cohort } & \multicolumn{2}{|c|}{$\begin{array}{l}\text { Patients without } \\
\text { disabilities }\end{array}$} & \multicolumn{2}{|c|}{$\begin{array}{l}\text { Patients with } \\
\text { disabilities }\end{array}$} & \multirow[t]{2}{*}{ p-value ${ }^{a}$} \\
\hline & No. $(\%)$ & Per 100,000 & No. $(\%)$ & Per 100,000 & No. $(\%)$ & Per $\mathbf{1 0 0 , 0 0 0}$ & \\
\hline Total MM patients & $4,090(100)$ & 36.8 & 3,281 (100) & 39.4 & $809(100)$ & 29.1 & $<0.001$ \\
\hline \multicolumn{8}{|l|}{ Age (yr) } \\
\hline$<65$ & $1,281(31.3)$ & 17.9 & $1,025(31.2)$ & 19.3 & $256(31.6)$ & 13.9 & $<0.001$ \\
\hline$\geq 65$ & $2,809(68.7)$ & 77.2 & $2,256(68.8)$ & 82.9 & $553(68.4)$ & 60.1 & $<0.001$ \\
\hline \multicolumn{8}{|l|}{ Sex } \\
\hline Male & $2,445(59.8)$ & 37.6 & $1,982(60.4)$ & 40.7 & $463(57.2)$ & 28.5 & $<0.001$ \\
\hline Female & $1,645(40.2)$ & 35.7 & $1,299(39.6)$ & 37.6 & $346(42.8)$ & 30.0 & $<0.001$ \\
\hline \multicolumn{8}{|l|}{ Income } \\
\hline Below poverty line (lowest) & $288(7.0)$ & 36.4 & $189(5.8)$ & 58.4 & $99(12.2)$ & 21.2 & $<0.001$ \\
\hline I & $653(16.0)$ & 32.9 & $528(16.1)$ & 35.9 & $125(15.5)$ & 24.3 & \\
\hline II & $610(14.9)$ & 31.7 & $483(14.7)$ & 33.1 & $127(15.7)$ & 27.4 & \\
\hline III & $899(22.0)$ & 35.9 & $708(21.6)$ & 36.7 & $191(23.6)$ & 33.2 & \\
\hline IV (highest) & $1,640(40.1)$ & 45.5 & $1,373(41.8)$ & 48.1 & $267(33.3)$ & 35.7 & \\
\hline \multicolumn{8}{|l|}{$\begin{array}{l}\text { MM-related complications } \\
\text { at the time of diagnosis }\end{array}$} \\
\hline Fracture & 744 (18.2) & & $605(18.4)$ & & 139 (17.2) & 17.2 & 0.406 \\
\hline Dialysis & 459 (11.2) & & $327(10.0)$ & & $132(16.3)$ & 16.3 & $<0.001$ \\
\hline
\end{tabular}

MM, multiple myeloma. a)Chi-square test. 
Table 2. Differences in the diagnosis of MM according to severity and type of disability

\begin{tabular}{|c|c|c|c|c|c|c|}
\hline & \multirow{2}{*}{\multicolumn{2}{|c|}{$\begin{array}{c}\text { MM diagnosis in } \\
\text { people with disabilities }\end{array}$}} & \multicolumn{4}{|c|}{ MM-related complications at the time of diagnosis } \\
\hline & & & \multicolumn{2}{|c|}{ Fracture } & \multicolumn{2}{|c|}{ Dialysis } \\
\hline & No. & Per 100,000 & No. $(\%)$ & p-value & No. $(\%)$ & p-value \\
\hline Total & 809 & 29.1 & $139(17.2)$ & & $132(16.3)$ & \\
\hline \multicolumn{7}{|l|}{ Severity of disability } \\
\hline Severe (grade $1,2,3$ ) & 258 & 23.6 & $42(16.3)$ & 0.639 & $69(26.7)$ & $<0.001$ \\
\hline Mild (grade 4, 5, 6) & 551 & 32.7 & $97(17.6)$ & & $63(11.4)$ & \\
\hline \multicolumn{7}{|l|}{ Type of disability } \\
\hline Physical & 506 & 29.3 & $100(19.8)$ & 0.139 & $58(11.5)$ & $<0.001$ \\
\hline Communication & 208 & 35.3 & $28(13.5)$ & & $24(11.5)$ & \\
\hline Intellectual or psychological & 23 & 7.5 & $3(13.0)$ & & $5(21.7)$ & \\
\hline Major internal organ & 72 & 47.6 & $8(11.1)$ & & $45(45.0)$ & \\
\hline
\end{tabular}

MM, multiple myeloma.

Table 3. Analysis of ASCT performance according to the presence, severity, and type of disability

\begin{tabular}{|c|c|c|c|c|}
\hline & \multirow{2}{*}{ All patients } & \multirow{2}{*}{$\frac{\text { Patients }<65 \mathrm{yr}}{\text { No. }(\%)}$} & \multicolumn{2}{|c|}{ Patients undergone ASCT } \\
\hline & & & No. $(\%)$ & p-value \\
\hline All patients & 4,090 & $1,281(31.3)$ & $544(42.5)$ & \\
\hline Patients without disabilities & 3,281 & $1,025(31.2)$ & $448(43.7)$ & 0.072 \\
\hline Patients with disabilities & 809 & $256(31.6)$ & $96(37.5)$ & \\
\hline \multicolumn{5}{|l|}{ Severity of disability } \\
\hline Severe (grade 1, 2, 3) & 258 & $107(41.5)$ & $29(27.1)$ & 0.004 \\
\hline Mild (grade 4, 5, 6) & 551 & $149(27.0)$ & $67(45.0)$ & \\
\hline \multicolumn{5}{|l|}{ Type of disability } \\
\hline Physical & 506 & $164(32.4)$ & $69(42.1)$ & 0.003 \\
\hline Communication & 208 & $37(17.8)$ & $17(45.9)$ & \\
\hline Intellectual or psychological & 23 & $18(78.3)$ & $6(33.3)$ & \\
\hline Major internal organ & 72 & $37(51.4)$ & $4(10.8)$ & \\
\hline
\end{tabular}

ASCT, autologous stem cell transplantation.

$62.3 \%)$, followed by communication disabilities $(n=588,712$, $21.2 \%)$, intellectual or psychological disabilities $(n=307,720$, $11.1 \%)$, and major internal organ disabilities $(n=151,102$, $5.4 \%$ ). People with intellectual or psychological disabilities had the youngest median age, at 33.6 years. Approximately half of people with an intellectual or psychological disability $(45.5 \%)$ had incomes below the poverty line (S3 Table).

\section{Diagnosis of MM}

Detailed information regarding the patients with $\mathrm{MM}$ is shown in Table 1 . The number of MM cases per 100,000 people was significantly lower in the disabled group than in the non-disabled group. The number of MM cases in the non- disabled group was highest among individuals with incomes below the poverty line, and the number of MM cases decreased with an increase in income. In contrast, in the disabled group, the lowest number of MM cases was observed among individuals with incomes below the poverty line, and the number of MM cases increased with an increase in income. There was no significant difference in the risk of fracture between patients with and without disabilities. However, patients with disabilities were more likely to start hemodialysis upon their MM diagnosis compared to patients without disabilities.

Table 2 summarizes the information pertaining to patients with $\mathrm{MM}$ and disabilities. The number of MM cases per 100,000 people was significantly lower in the group with 
A
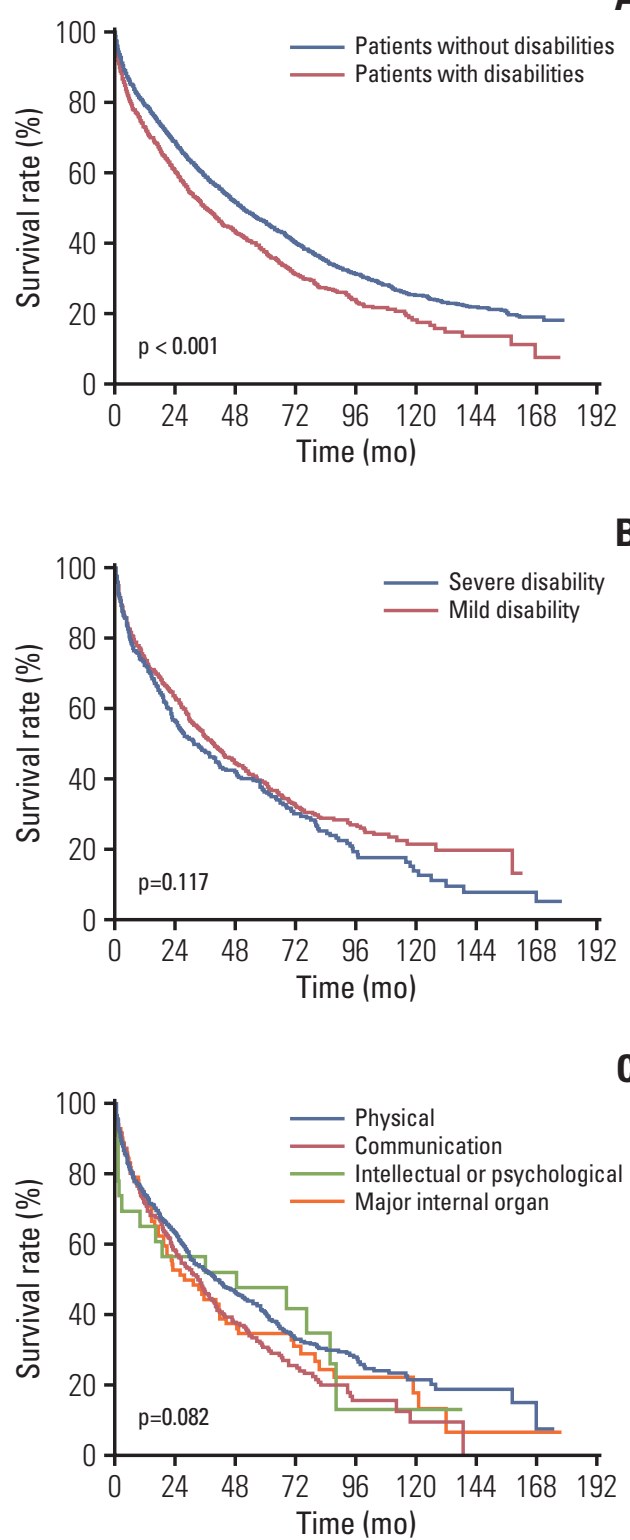

Fig. 3. Overall survival comparison by presence or absence of disability (A), severity of disability (B), type of disability (C).

severe disabilities than in the group with mild disabilities $(\mathrm{p}<0.001)$. The prevalence of MM was much lower in the subgroup of people with intellectual or psychological disabilities, relative to the other types. Patients with severe disabilities received dialysis more frequently than patients with mild disabilities, and dialysis was also more common among patients with intellectual or psychological disabilities and major internal organ disabilities than among patients with physical disabilities and communication disabilities. There was no significant difference in the frequency of fracture according to severity of the disability, although fractures were slightly more common among patients with physical disabilities and relatively less common for the other disability types.

\section{Analysis of treatment behavior}

Among the 1,281 patients with $\mathrm{MM}$ at the age of $<65$ years, 544 patients $(42.5 \%)$ received ASCT. The proportion of patients who receive ASCT was slightly lower in the group with disabilities than in the group of patients without disabilities (37.5\% vs. $43.7 \%, \mathrm{p}=0.072$ ) (Table 3). Twenty-nine patients $(27.1 \%)$ with severe disabilities received ASCT, which was significantly lower than the rate of $45.0 \%$ (67 patients) among patients with mild disabilities ( $\mathrm{p}=0.004)$. Among the various disability types, the lowest rates of ASCT were observed in the group with major internal organ disabilities and the group with intellectual or psychological disabilities. However, no significant differences with regard to the use of novel therapeutic agents were observed between patients with and without disabilities (S4 Table).

\section{Survival analysis}

The median overall survival (OS) of all MM patients was 48.3 months. After adjusting for sociodemographic characteristics, we observed a higher mortality risk among patients with MM and disabilities (median OS, 36.8 months) than among patients with MM but without disabilities (median OS, 51.2 months) (Fig. 3A). Among patients with disabilities, the risk of death was slightly higher among patients with severe disabilities, although this difference was not statistically significant (median OS, 31.2 months vs. 38.9 months; $\mathrm{p}=0.117$ ) (Fig. 3B). The median OS was markedly shorter in the major internal organ disability group, although the association between disability type and OS was not statistically significant (median OS, 41.0 months for physical disabilities, 33.9 months for communication disabilities, 48.2 months for intellectual or psychological disabilities, and 29.1 months for major internal organ disabilities; $\mathrm{p}=0.082$ ) (Fig. 3C).

\section{Discussion}

The prognosis of MM has recently improved, although advanced therapies are not equally available to all patients. This is partially related to the treatment of malignant diseases generally involving considerable toxicity, which means that patients must be physically and mentally able to tolerate 
any given treatment. Moreover, the development of novel treatments has resulted in a steady increase in the cost of treating MM. For example, an American report has confirmed that improvements in MM therapy have increased the survival rate, but with a corresponding increase in the total cost of treating MM [13]. This socioeconomic burden can lead to disparities in treatment approaches between patient groups or between countries. In this context, recent studies have shown that developed countries have improved MM mortality rates, while low- or medium-income countries have low rates of MM diagnosis and delayed introduction of advanced therapies, including novel agents and ASCT [14].

Our results indicate that people with disabilities were less likely to be diagnosed with MM than people without disabilities. Moreover, patients with disabilities were more likely to experience MM-related renal failure at an early phase of the disease. The causes of delayed or missed MM diagnoses in people with disabilities are unclear, but several reasons can be speculated. People with disabilities sometimes cannot recognize or express their symptoms properly, because their underlying medical problems mask the manifestations of MM. Moreover, visiting professional medical institutions can be a challenge if one's movement is restricted due to a physical disability.

Another important reason is the low socioeconomic status (SES) of people with disabilities. Disabilities are known to be directly associated with a low employment rate and low economic activity [15-19]. Our study provides additional evidence indicating that people with disabilities are more likely to have low incomes. The relationship between low SES and delayed diagnosis of cancers has also been reported in previous studies. In the United States, the effect of insurance status and ethnicity on cancer diagnoses of 12 sites was analyzed. Uninsured or Medicaid-insured patients and black or Hispanic patients were more commonly diagnosed at advanced stages [20]. Similar results were obtained in studies of breast, colorectal, and prostate cancer [21]. Although there have been few studies of patients with hematologic malignancies, a study of the Danish registry of patients with nonHodgkin lymphoma found that the lymphoma was discovered at a higher stage in those with a lower SES than in those with a higher SES [22]. Therefore, it can be assumed that the relatively low SES levels of the group with disabilities affected the delay or absence of a diagnosis.

Nevertheless, the diagnosis rates of MM in people without disabilities were not significantly different between individuals with incomes below the poverty line and individuals in the upper-income groups. It is presumed that Korea's medical aid program, which provides free basic medical services to people in the lowest income bracket, prevents the deterioration of medical service utilization due to economic problems. On the other hand, among people with disabilities, the lowest diagnosis rate was observed among individuals with incomes below the poverty line. This means that the current economic support is not sufficient to overcome the barriers to accessing healthcare that is faced by low-income people with disabilities, which highlights the importance of more comprehensive support, which should target a wide spectrum including physical mobility, the provision of care, and improved of awareness regarding the health problems of disabled people.

The diagnosis rate of $\mathrm{MM}$ also varied according to the type of disability, with the lowest diagnosis rate observed among people with intellectual or psychological disabilities. Many of the causes of intellectual or psychological disabilities are diagnosed at a young age, with patients having a relatively short average life span that can preclude survival until a diagnosis of cancer $[23,24]$. Meanwhile, another report has also indicated that a number of health problems can affect people with intellectual or psychological disabilities, although those problems are frequently underdiagnosed [25]. Our results demonstrated that the ages and MM diagnosis rates of people with intellectual or psychological disabilities were lower than those with other types of disabilities, but these patients were diagnosed at relatively late stages, based on the need for dialysis treatment. The poor economic status of patients with MM plus intellectual or psychological disabilities may also interfere with their diagnosis and treatment. In addition, patients with intellectual or psychological disabilities may not correctly perceive or express their symptoms, which may also delay diagnosis and treatment. Thus, special efforts are needed to obtain accurate information from these patients and to appropriately communicate appropriately regarding the treatment that they should receive. For example, experts recommend that interactions with individuals with intellectual disabilities involve plenty of time, simple words, patience, and repeated explanations [26].

Disabilities also affect the treatment patterns of MM. Among patients who are $<65$ years old, the standard treatment is induction chemotherapy followed by high-dose chemotherapy and ASCT [27-29], although the high toxicity of this approach, and the potential for severe adverse events, may limit its use in patients with a favorable general health status. For example, we found that patients with MM plus disabilities had a lower rate of receiving ASCT than patients without disabilities, with the lowest rates of ASCT observed for patients with intellectual, psychological, and major internal organ disabilities. The low rate of ASCT use among patients with major internal organ disabilities is reasonable, as their disabilities can inherently increase the risk of complications after ASCT. However, the low rate of ASCT among patients with intellectual or psychological disabilities cannot be solely explained based on medical factors. It may be difficult to educate these patients appropriately and to ensure 
that they consent and are committed to the treatment. Furthermore, the high costs of ASCT inherently conflict with the low economic status of people with intellectual or psychological disabilities. Moreover, people with intellectual or psychological disabilities often depend heavily on caregivers, including family members, suggesting that the passive attitude of caregivers could influence the treatment approach for patients with MM plus intellectual or psychological disabilities.

OS rates were also inferior in cases of MM patients with disabilities compared to patients without disabilities. The causal relationship between disability and MM-associated death cannot be evaluated because the cause of death of each patient was not determined individually. Nevertheless, we can assume why mortality rates are high in disabled groups based on observed phenomena. In addition to the possibility that the disability itself may have had a negative impact on their medical conditions, the delayed diagnosis and less intensive treatment may also contribute to their poor survival rates. Furthermore, the relatively low SES levels of patients with disabilities also may be associated with the inferior prognosis of MM. Several studies have shown that the prognosis of malignant diseases, including blood cancers, is related to patients' SES. In a study based on a national cohort of Sweden, Kristinsson et al. [30] reported that selfemployed, blue-collar, and retired patients with acute myeloid leukemia or MM were generally associated with high mortality rates. Danish researchers have also shown a higher mortality rate among non-Hodgkin's lymphoma patients in non-employment status, disability pension recipients, lowincome brackets, and single patients [22]. These authors also demonstrated that these poor socioeconomic factors were associated with low access to treatment, including radiation therapy. In compiling this data and the results of the preceding studies, it could be hypothesized that the low SES of disabled patients interferes with access to treatment, and that inappropriate management of MM exacerbates their prognosis. Proper management of MM patients with disabilities requires a complex assessment, including not only the patient's medical problems but also their living environments and economic situations, and individualized, multidisciplinary approach should be made in each case to ensure that these patients receive the best care.

The present study has several limitations. First, the use of population-based registries precluded more detailed analyses regarding the degree of disability, disease status, treatment approaches, and prognosis. We could not directly analyze the causal relationships between these factors and instead presented only hypotheses based on the various results suggesting their relevance. Therefore, additional assessments such as patient-control studies are needed for a proper assessment of the impacts of various factors related to disabilities on MM treatments and prognoses. We also excluded patients who had a disability after receiving MM diagnosis from the group of MM patients with disabilities, although in reality some of these cases may not have been due to MM. Furthermore, the use of national health insurance data excludes patients who pay directly for their treatment or who participate in clinical trials. Therefore, our findings are not completely representative of treatment approaches for patients with MM, although we believe that ours is the first study to examine the effects of disabilities on the diagnosis and treatment of MM.

In conclusion, the present study revealed that Korean patients with disabilities face various barriers to the effective diagnosis and treatment of MM. These barriers may limit the benefit of novel therapies to patients with disabilities relative to those without disabilities. Therefore, we suggest that economic support must be combined with social support for people with disabilities, including an improved awareness of their disabilities and better provision of general care.

\section{Electronic Supplementary Material}

Supplementary materials are available at Cancer Research and Treatment website (https:// www.e-crt.org).

\section{Conflicts of Interest}

Conflict of interest relevant to this article was not reported.

\section{References}

1. Jung KW, Won YJ, Kong HJ, Lee ES; Community of Population-Based Regional Cancer Registries. Cancer statistics in Korea: incidence, mortality, survival, and prevalence in 2015. Cancer Res Treat. 2018;50:303-16.

2. Park HJ, Park EH, Jung KW, Kong HJ, Won YJ, Lee JY, et al. Statistics of hematologic malignancies in Korea: incidence, prevalence and survival rates from 1999 to 2008. Korean J Hematol. 2012;47:28-38.

3. Auner HW, Szydlo R, Hoek J, Goldschmidt H, Stoppa AM, Morgan GJ, et al. Trends in autologous hematopoietic cell transplantation for multiple myeloma in Europe: increased use and improved outcomes in elderly patients in recent years. 
Bone Marrow Transplant. 2015;50:209-15.

4. Brenner H, Gondos A, Pulte D. Recent major improvement in long-term survival of younger patients with multiple myeloma. Blood. 2008;111:2521-6.

5. Kumar SK, Dispenzieri A, Lacy MQ, Gertz MA, Buadi FK, Pandey S, et al. Continued improvement in survival in multiple myeloma: changes in early mortality and outcomes in older patients. Leukemia. 2014;28:1122-8.

6. Korean Statistical Information Service, Korea Statistical Information Services. National survey on persons with disabilities. Seoul: Korea Institute for Health and Social Affairs; 2011.

7. Jung KW, Park S, Kong HJ, Won YJ, Lee JY, Seo HG, et al. Cancer statistics in Korea: incidence, mortality, survival, and prevalence in 2009. Cancer Res Treat. 2012;44:11-24.

8. Jee SH, Sull JW, Park J, Lee SY, Ohrr H, Guallar E, et al. Bodymass index and mortality in Korean men and women. N Engl J Med. 2006;355:779-87.

9. Kim YI, Kim SY, Cho SJ, Park JH, Choi IJ, Lee YJ, et al. Longterm metformin use reduces gastric cancer risk in type 2 diabetics without insulin treatment: a nationwide cohort study. Aliment Pharmacol Ther. 2014;39:854-63.

10. Seong SC, Kim YY, Khang YH, Park JH, Kang HJ, Lee H, et al. Data resource profile: The National Health Information Database of the National Health Insurance Service in South Korea. Int J Epidemiol. 2017;46:799-800.

11. Korean Statistical Information Service. Number of registered disabled people [Internet]. Daejeon: Statistics Korea; c2017 [cited 2018 Apr 26]. Available from: http://kosis.kr/index/ index.do.

12. Yoon TH, Lee SY, Kim CW, Kim SY, Jeong BG, Park HK. Inequalities in medical care utilization by South Korean cancer patients according to income: a retrospective cohort study. Int J Health Serv. 2011;41:51-66.

13. Fonseca R, Abouzaid S, Bonafede M, Cai Q, Parikh K, Cosler $\mathrm{L}$, et al. Trends in overall survival and costs of multiple myeloma, 2000-2014. Leukemia. 2017;31:1915-21.

14. Cowan AJ, Allen C, Barac A, Basaleem H, Bensenor I, Curado $\mathrm{MP}$, et al. Global burden of multiple myeloma: a systematic analysis for the Global Burden of Disease Study 2016. JAMA Oncol. 2018;4:1221-7.

15. Fujiura GT, Yamaki K. Analysis of ethnic variations in developmental disability prevalence and household economic status. Ment Retard. 1997;35:286-94.

16. Smith JP. Healthy bodies and thick wallets: the dual relation between health and economic status. J Econ Perspect. 1999;13: 144-66.

17. Emerson E. Mothers of children and adolescents with intellectual disability: social and economic situation, mental health status, and the self-assessed social and psychological impact of the child's difficulties. J Intellect Disabil Res. 2003;47(Pt
4-5):385-99.

18. Stabile M, Allin S. The economic costs of childhood disability. Future Child. 2012;22:65-96.

19. Kavanagh AM, Krnjacki L, Aitken Z, LaMontagne AD, Beer A, Baker E, et al. Intersections between disability, type of impairment, gender and socio-economic disadvantage in a nationally representative sample of 33,101 working-aged Australians. Disabil Health J. 2015;8:191-9.

20. Halpern MT, Ward EM, Pavluck AL, Schrag NM, Bian J, Chen AY. Association of insurance status and ethnicity with cancer stage at diagnosis for 12 cancer sites: a retrospective analysis. Lancet Oncol. 2008;9:222-31.

21. Byers TE, Wolf HJ, Bauer KR, Bolick-Aldrich S, Chen VW, Finch JL, et al. The impact of socioeconomic status on survival after cancer in the United States: findings from the National Program of Cancer Registries Patterns of Care Study. Cancer. 2008;113:582-91.

22. Frederiksen BL, Dalton SO, Osler M, Steding-Jessen M, de Nully Brown P. Socioeconomic position, treatment, and survival of non-Hodgkin lymphoma in Denmark: a nationwide study. Br J Cancer. 2012;106:988-95.

23. Coppus AM. People with intellectual disability: what do we know about adulthood and life expectancy? Dev Disabil Res Rev. 2013;18:6-16.

24. Patja K, Iivanainen M, Vesala H, Oksanen H, Ruoppila I. Life expectancy of people with intellectual disability: a 35-year follow-up study. J Intellect Disabil Res. 2000;44(Pt 5):591-9.

25. Haveman M, Perry J, Salvador-Carulla L, Walsh PN, Kerr M, Van Schrojenstein Lantman-de Valk H, et al. Ageing and health status in adults with intellectual disabilities: results of the European POMONA II study. J Intellect Dev Disabil. 2011;36:49-60.

26. Riddle I, Romelczyk S, Sparling E. Effective communication for health care providers: a guide to caring for people with disabilities. Newark, DE: Center for Disabilities Studies, University of Delaware; 2011.

27. Rajkumar SV. Multiple myeloma: 2016 update on diagnosis, risk-stratification, and management. Am J Hematol. 2016;91: 719-34.

28. Kumar SK, Callander NS, Alsina M, Atanackovic D, Biermann JS, Castillo J, et al. NCCN guidelines insights: multiple myeloma, version 3.2018. J Natl Compr Canc Netw. 2018;16:11-20.

29. Cavo M, Terpos E, Bargay J, Einsele H, Cavet J, Greil R, et al. The multiple myeloma treatment landscape: international guideline recommendations and clinical practice in Europe. Expert Rev Hematol. 2018;11:219-37.

30. Kristinsson SY, Derolf AR, Edgren G, Dickman PW, Bjorkholm $\mathrm{M}$. Socioeconomic differences in patient survival are increasing for acute myeloid leukemia and multiple myeloma in sweden. J Clin Oncol. 2009;27:2073-80. 